\title{
Cell therapy for osteoarthritis
}

\begin{abstract}
Osteoarthritis (OA) also known as degenerative joint disease or degenerative arthritis is the most common chronic condition of the joints. It mainly damages the articular cartilage which results in pain, swelling and stiffness around the joints. It is the leading cause of disability and pain. Current therapeutic strategies are expensive and they are capable of eradicating only the symptoms of OA instead of reversing the damage caused by it. The aim of this review is to introduce the latest stem cell advances as a novel and effective therapeutics for the treatment of OA. One of the most effective stem cell source is mesenchymal stem cells (MSCs) derived from bone marrow as well as from adipose tissues. Other cell sources which have the capability of becoming a novel treatment for OA such as induced pluripotent stem cells (iPSs), embryonic stem cells (ESCs) and extracellular vesicles derived from MSCs have also been highlighted in this review.
\end{abstract}

Volume 3 Issue 2 - 2017

\author{
Minal Thacker,' Feng-Huei Lin ${ }^{1,2}$ \\ 'Institute of Biomedical engineering, National Taiwan University, \\ Taiwan \\ 2Institute of Biomedical Engineering \& Nanomedicine, National \\ Health Research Institute, Taiwan
}

Correspondence: Feng-Huei Lin, Distinguished Professor, Department of Biomedical Engineering, National Taiwan University, Taipei, Taiwan, Tel 886928260400 , Email double@ntu.edu.tw

Received: June 26, 2017 | Published: August 02, 2017

Keywords: osteoarthritis, stem cells, cartilage regeneration, mscs, ipss, escs

\section{Introduction}

Degenerative joint disease or degenerative arthritis commonly known as Osteoarthritis (OA) is the most common chronic condition of the joints which affects all ages and genders but possess a greater risk in elderly and obese people. OA can affect any joint, but more prevalently affects the knee and hip. ${ }^{1}$ It is the fourth leading cause of pain and disability worldwide. ${ }^{2}$

OA is characterized mainly by loss or damage of articular cartilage. ${ }^{3}$ Cartilage acts as a soft cushion between the bones and provides a smooth surface for joint movement. The loss of this cushion would cause pain and swelling. ${ }^{4}$ In addition remodeling of bone joints also occurs which causes chips of bones to float around in the joints and also develop growth called spurs which leads to the inflammatory action followed by the production of cytokines which further damages the cartilage and worsens the pain. ${ }^{5}$ There are several factors that contribute to the development of OA such as weight, injury or overuse of joints, genetic factors but most important of it all is the age factor. As the age progresses the extracellular matrix (ECM) surrounding the primary cells of cartilage called chondrocytes alters their structural property which eventually causes the cartilage to bear less mechanical stress. ${ }^{6,7}$

The severity and the affected area in OA vary and so are the symptoms, the most common being swelling, pain and stiffness which makes the usual daily chores difficult to perform. ${ }^{8}$ Conventional medical strategies for treating $\mathrm{OA}$ aimed at only managing the symptoms instead of eradicating the disease. Non-surgical treatments such as physiotherapy, pain relievers and anti -inflammatory drugs and weight loss have a short term relief. ${ }^{9}$ On the other hand, surgical treatment such as total knee replacement (TKR) or hip replacement is widely used to treat knee OA and Hip OA respectively, but often followed by complications such as infections which again requires a hospital visit. Some of the patients still continue to have knee pain post-surgery. ${ }^{10}$ However, in order to overcome the drawbacks of the conventional method, novel strategies using stem cells is storming through the field of therapeutics over the past few decades showing promising results for the treatment of OA.

\section{Cell based therapy}

Cell based therapy emerged in 1980 and it has been evolved since then (Figure 1). Autologous chondrocyte implantation (ACI) is the most conventional cell based treatment that evolved over past 20 years with a high success rate. However, it constricts itself to the site of cartilage damage and was an exclusion to the generalized OA treatment. ${ }^{11}$ The limitation of ACI for the OA treatment demanded the emergence of another cell source. Stem cells proved to be a promising candidate for the cartilage regeneration. ${ }^{12}$ Stem cells are the undifferentiated cells that are capable of differentiating into specialized cells such as osteocytes, chondrocytes, adipocytes etc. Stem cells in addition are also capable of downregulating inflammation by releasing important cytokines and certain proteins which are essential in overcoming OA. ${ }^{13}$

Other two types of stem cells such as embryonic stem cells (ESCs) and induced pluripotent stem cells (iPSCs) were also examined to be a potential candidate for cartilage regeneration for combating OA. Both ESCs and iPSCs are pluripotent in nature and can differentiate into any other cell type including chondrocytes. ${ }^{14}$ In 2012 Wei et al. generated iPSCs from osteoarthritic chondrocytes and induced these cells into chondrocytic differentiation. ${ }^{15}$ Though both ESCs and iPSCs have a potential to differentiate into cartilage cells to treat OA, certain limitations exist such as both the cell types are at risk of forming teratoma and immunogenicity. ${ }^{16}$

The most ideal amongst different types of stem cells for combating $\mathrm{OA}$ are mesenchymal stem cell (MSC). These can be derived from various sources such as bone marrow, adipose tissue, umbilical cord, Wharton's jelly and so on. MSCs are widely studied for OA treatment as it expresses certain enzymes such as growth factors, cytokines and are also capable of exerting anti-inflammatory activity. ${ }^{17,18}$ Recently Davatchi et al. ${ }^{19}$ studied 5year follow up of three patients of knee OA. The authors injected autologous bone marrow derived MSCs into the worst knee of patients aged 57, 65 and 54years. The study examined that the implanted knee was better than at the baseline while the better knee became the worst knee by the end of 5years. The article concluded that in long term follow up earlier transplantation would give a better result for advanced stage of OA. ${ }^{19}$ Subsequently 
Mehrabani et al. treated a middle aged 47years old nomad woman suffering from a severe right knee OA with an intra- articular injection of bone marrow- derived MSCs. The follow up after 2 months posttransplantation revealed the improved state of the affected knee as her walking distance increased, she could climb more number of stairs and most importantly magnetic resonance images (MRI) unveil the repaired tissue extension over the subchondral bone. Therefore, the authors proposed that MSC transplantation for OA is an easy and convenient technique resulting in pain relief, improved cartilage quality without surgery and minimized donor site morbidity. ${ }^{20}$ The safety and efficacy of administrating MSCs in OA patients have been proved by various preclinical and clinical trials, but most of the studies were limited to single-dose, single- pilot studies. ${ }^{21,22}$ Therefore, for more randomized and controlled clinical trials, Gupta et al. ${ }^{23}$ studied the efficiency of differentiation of adult human bone marrow-derived, cultured, pooled, allogeneic mesenchymal stromal cells (Stempeucel) into the chondrogenic lineage. Further they carried out the preclinical trial by injecting these cells intra-articularly into the knee joints of OA induced rats. Post the preclinical trials a clinical study was established in order to find the doseage of Stempeucel in knee joint OA patients. The authors concluded that the stempeucel successfully differentiated into chondrocytes in vitro, further it greatly reduced pain and also repaired the articular cartilage in OA induced rats in preclinical studies. Finally during the clinical trial they concluded that a 25 million cell dose might be the most effective for reducing pain in OA patients..$^{23}$ Despite of the phenomenal ability of MSCs differentiating into chondrocytes there have been certain fatal risks recorded post transplantation. Therefore, Famian et al. ${ }^{24}$ examined Wharton's jelly derived stem cell conditioned medium (WJSCs$\mathrm{CM})$ for the enhanced gene expression profile by chondrocytes. The authors suggested that WJSCs-CM have the ability to enhance the cartilage specific genes and can be used as a potential factor for cartilage regeneration. ${ }^{24}$

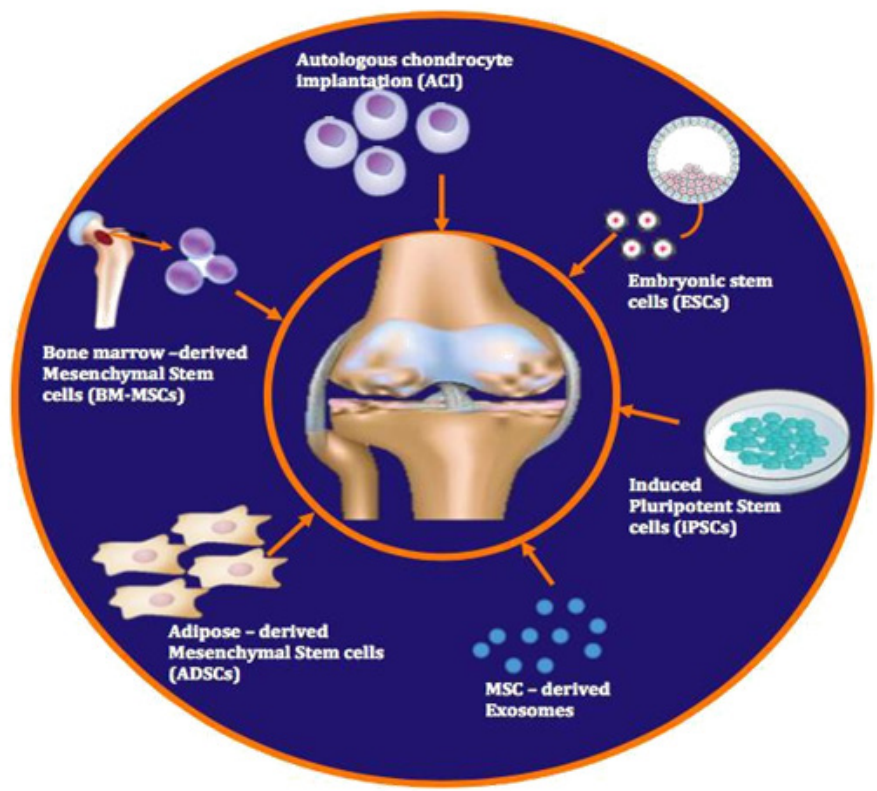

Figure I Schematic representation of various cell-based therapy for osteoarthritis.

Adipose tissue is another perfect source for obtaining MSCs and the cells obtained from adipose tissue are called adipose tissue derived mesenchymal stem cells (ASCs). They are capable of differentiating into bones and cartilage. ${ }^{25}$ Pagani et al. ${ }^{26}$ carried out a study wherein, human MSCs derived from bone marrow (hBMSCs) and adipose tissue (hADSCs) were compared based on their behavior in OA microenvironment. Thus, they concluded that hADSCs have a better chondrogenic potential in OA microenvironment. ${ }^{26}$

Mei et al. ${ }^{27}$ studied the treatment of anterior cruciate ligament transection (ACLT) induced rat OA model by intra-articular injections of culture-expanded allogenic adipose tissue-derived stem cells (ADSCs). The authors revealed that cartilage degeneration in rat OA model was put on halt with intra-articular injections of ADSCs; it also shielded the chondrocytes from the inflammatory factor. ${ }^{27}$ Recently, Parrilli et al. $^{28}$ focused on subchondral bone in order to treat OA. They injected adipose derived stromal cells into the joint to prevent subchondral bone damage after the onset of OA in rabbit. They showed that ASCs can promote cartilage regeneration as well as attenuate the increased bone turnover which is a characteristic of $\mathrm{OA}^{28}$ Phase I clinical trial was conducted by Pers et al. ${ }^{29}$ to treat severe knee OA using ADSC. 18 patients with severe knee OA were injected with intra-articular injections of autologous ASCs, the follow up after 6months indicated that ASCs can be a potential alternative for the treatment of severe knee OA. ${ }^{29}$

Extracellular vesicle (EV) is a small vesicle enclosed by a phospholipid bilayer. It gained a lot of attention as it provides an easy way of cell-to-cell communication which helps in exchanging functional molecules of one cell to another in its vicinity. Almost all types of cells including MSCs release these vesicles. There are very few studies that have shown the therapeutic effect of MSC derived EVs in combating OA. These studies showed that MSC-EVs are capable of cartilage regeneration. ${ }^{17,30}$ The use of EVs as a therapeutic agent for treating OA still needs to be investigated further.

\section{Conclusion}

In this review the current progress in cell therapy for combating OA have been highlighted. The conventional treatment for OA is costly and is effective only in reducing the symptoms but not actually stopping or reversing the damage caused by it. As it is the fourth leading cause of disability, its rapid progression necessitates a novel and safe therapeutic approach. Stem cells, in particular MSCs derived from bone marrow and adipose tissue have a great potential in reversing the cartilage damage due to OA. However, studies have shown that hADSCs holds a greater chondrogenic potential in inflammatory environment such as OA. However, the number of clinical trials and long term follow ups in cell therapy needs to be increased to provide a fruitful treatment for OA. Subsequently, a wellplanned research is required to study the potential of MSC derived EVs for combating OA.

\section{Acknowledgements}

None.

\section{Conflict of interest}

The author declares no conflict of interest.

\section{References}

1. Uth K, Trifonov D. Stem cell application for osteoarthritis in the knee joint: A mini review. World J Stem Cells. 2014;6(5):629-636. 
2. Neogi T. The epidemiology and impact of pain in osteoarthritis. Osteoarthritis Cartilage. 2013;21(9):1145-1153.

3. Ondresik M, Azevedo Maia FR, da Silva Morais A, et al. Management of Knee Osteoarthritis. Current Status and Future Trends. Biotechnol Bioeng. 2017;114(4):717-739.

4. Litwic A, Edwards M, Dennison E, et al. Epidemiology and Burden of Osteoarthritis. Br Med Bull. 2013;105:185-199.

5. Yubo M, Yanyan L, Li L, et al. Clinical efficacy and safety of mesenchymal stem cell transplantation for osteoarthritis treatment:A metaanalysis. PLoS One. 2017;12(4):e0175449.

6. Freitag J, Bates D, Boyd R, et al. Mesenchymal stem cell therapy in the treatment of osteoarthritis: reparative pathways, safety and efficacy - a review. BMC Musculoskeletal Disorders. 2016;17:230.

7. Atesok K, Fu FH, Sekiya I, et al. Stem cells in degenerative orthopaedic pathologies: effects of aging on therapeutic potential. Knee Surg Sports Traumatol Arthrosc. 2017;25(2):626-636.

8. Zhang W, Ouyang H, Dass C, et al. Current research on pharmacologic and regenerative therapies for osteoarthritis. Bone Res. 2016;1(4):15040.

9. Girolamo L, Kon E, Filardo G, et al. Regenerative approaches for the treatment of early OA. Knee Surg Sports Traumatol Arthrosc. 2016;24(6):1826-1835.

10. Bhatia D, Bejarano T, Novo M. Current interventions in the managemen of knee osteoarthritis. J Pharm Bioallied. 2013;5(1):30-38.

11. Burke J, Hunter M, Kolhe R, et al. Therapeutic potential of mesenchymal stem cell based therapy for osteoarthritis. Clin Transl Med. 2016;5(1):27.

12. Diekman B, Guilak F. Stem cell-based therapies for osteoarthritis: Challenges and opportunities. Curr Opin Rheumatol. 2013;25(1):119-126.

13. Baraniak P, McDevitt T. Stem cell paracrine actions and tissue regeneration. Regen Med. 2010;5(1):121-143.

14. Chang $\mathrm{Y}-\mathrm{H}$, Liu $\mathrm{H}-\mathrm{W}, \mathrm{Wu} \mathrm{K}-\mathrm{C}$, et al. Mesenchymal Stem Cells and Their Clinical Applications in Osteoarthritis. Cell Transplantation. 2016;25(5):937-950.

15. Wei Y, Zeng W, Wan R, et al. Chondrogenic differentiation of induced pluripotent stem cells from osteoarthritic chondrocytes in alginate matrix. European cells and materials. 2012;23:1-12.

16. Pak J, Lee JH, Park KS, et al. Regeneration of Cartilage in Human Knee Osteoarthritis with Autologous Adipose Tissue-Derived Stem Cells and Autologous Extracellular Matrix. Biores Open Access. 2016;5(1):192200 .

17. Ruiz M, Cosenza S, Maumus M, et al. Therapeutic application of mesenchymal stem cells in osteoarthritis. Expert Opin Biol Ther. 2016;16(1):33 -42 .
18. Pers YM, Ruiz M, Noel D, et al. Mesenchymal stem cells for the management of inflammation in osteoarthritis: state of the art and perspectives. Osteoarthritis and Cartilage. 2015;2(11):2027-2035.

19. Davatchi F, Abdollahi BS, Mohyeddin M, et al. Mesenchymal stem cell therapy for knee osteoarthritis:5 years follow-up of three patients. Int $J$ Rheum Dis. 2016;19(3):219-225.

20. Mehrabani D, Mojtahed Jaberi F, Zakerinia M, et al. The Healing Effect of Bone Marrow-Derived Stem Cells in Knee Osteoarthritis:A Case Report. World J Plast Surg. 2016;5(2):168-174.

21. Li M, Luo X, Lv X, et al. In vivo human adipose-derived mesenchymal stem cell tracking after intra-articular delivery in a rat osteoarthritis model. Stem Cell Research \& Therapy. 2016;7(1):160.

22. Koh YG, Jo SB, Kwon OR, et al. Mesenchymal Stem Cell Injections Improve Symptoms of Knee Osteoarthritis. Arthroscopy. 2013;29(4):748755 .

23. Gupta P, Chullikana A, Rengasamy M, et al. Efficacy and safety of adult human bone marrow-derived, cultured, pooled, allogeneic mesenchymal stromal cells (Stempeucel $\left.{ }^{\circledR}\right)$ : preclinical and clinical trial in osteoarthritis of the knee joint. Arthritis Research and Therapy. 2016;18(1):301.

24. Famian MH, Saheb SM, Montaseri A. Conditioned Medium of Wharton's Jelly Derived Stem Cells Can Enhance the Cartilage Specific Genes Expression by Chondrocytes in Monolayer and Mass Culture Systems. Adv Pharm Bull. 2017;7(1):123-130.

25. Pak J, Jung Hun Lee, Kwang Seung Park, et al. Current use of autologous adipose tissue- derived stromal vascular fraction cells for orthopedic applications. Journal of Biomedical Science. 2017;24:9.

26. Pagani S, Borsari V, Veronesi F, et al. Increased Chondrogenic Potential of Mesenchymal Cells From Adipose Tissue Versus Bone Marrow-Derived Cells in Osteoarthritic In Vitro Models. J Cell Physiol. 2017;232(6):1478-1488.

27. Mei L, Shen B, Ling P, et al. Culture-expanded allogenic adipose tissuederived stem cells attenuate cartilage degeneration in an experimental rat osteoarthritis model. PLoS One. 2017;12(4):e0176107.

28. Parrilli A, Giavaresi G, Ferrari A, et al. Subchondral bone response to injected adipose-derived stromal cells for treating osteoarthritis using an experimental rabbit model. Biotechnic \& Histochemistry. 2017;92(3):201-211.

29. Pers YM, Rackwitz L, Ferreira R, et al. Adipose Mesenchymal Stromal Cell-Based Therapy for Severe Osteoarthritis of the Knee:A Phase I Dose-Escalation Trial. Stem Cells Translational Medicine. 2016;5(7):847-856.

30. Cosenza S, Ruiz M, Maumus M, et al. Pathogenic or Therapeutic Extracellular Vesicles in Rheumatic Diseases: Role of Mesenchymal Stem Cell-Derived Vesicles. Int J Mol Sci. 2017;18(4):889. 